\title{
Studies of the factorisation of proton densities in van der Meer scans and its impact on precision luminosity measurements for CMS
}

\author{
Peter Major* (on behalf of the CMS Collaboration) \\ Eötvös Loránd University, Budapest \\ MTA-ELTE Lendület Particle and Nuclear Physics Group \\ E-mail: pmajor@cern. ch ${ }^{\dagger}$
}

\begin{abstract}
The factorisability of the transverse proton density functions in $x$ and $y$ is assumed in the analysis of single-plane van der Meer (vdM) scans for absolute luminosity calibration. A correction to the calibration constants for nonfactorisation effects in the proton density is then determined. The precision of the evaluation of this correction is one of the dominant sources of systematic uncertainty for the Run 2 (2015-2018) luminosity measurement in CMS. The vdM calibration technique and the methods used to estimate the bias due to the factorisation assumption are discussed.
\end{abstract}

European Physical Society Conference on High Energy Physics - EPS-HEP2019 -

10-17 July, 2019

Ghent, Belgium

\footnotetext{
* Speaker.

$\dagger$ The author wishes to thank for their support the Hungarian Academy of Sciences "Lendület" (Momentum) Program (LP 2015-7/2015) and the National Research, Development and Innovation Office of Hungary (K 124845 and K 128713).
} 


\section{Introduction}

The CMS [1] experiment at LHC measures luminosity using several subdetectors (luminometers) [2,3]. The van der Meer (vdM) method [4] is employed to calibrate the luminometers and hence obtain the absolute luminosity scale. This method assumes that the proton density of the bunches factorise in the $x$ and the $y$ planes. In general this assumption holds only approximately which introduces a bias to the measured luminosity. In this paper, I shortly introduce the current methods used in CMS to gauge the size of the bias and derive a corresponding correction and uncertainty.

\section{Beam-imaging analysis}

The beam-imaging scan [5] consists of two pairs of special vdM scans. During a given scan one of the beams is at a fixed position while the other scans it in either the $x$ or the $y$ plane in 19 equidistant steps as seen in Fig.1. The scans are performed in both $x$ and $y$ and for both beams stationary. During this procedure primary-vertex data are recorded gated on five bunch pairs. The vertex data are then used to fit different models of the two-dimensional proton density functions of the bunches. An example for the residuals ((data-fit)/uncertainty) is seen on Fig. 2. These models are built as the sum of 2D Gaussian functions. Currently the most complex model used has three components, where the third component is narrow with respect to the first two and has a negative weight. The correction values are derived from simulated vdM scans. The bias of the method is evaluated using simulated beam-imaging scan analyses with randomly generated bunch density profiles (Fig.3). Figure 4 shows the compatibility of the derived corrections for the different bunches and models in 2017.

\section{Offset scan analysis}

Offset scans are auxiliary measurements and are analysed together with a normal vdM scan. These scans are performed the same way as vdM scans albeit the two beams are separated in the nonscanning direction to a distance of $2.5 \sigma$. Under the assumption that the proton density of the bunches does not change during the scans, the offset scans sample the tails of the beam-beam convolution function (Fig.5). The data representing a sampling of this convolution function are then fit with models built as the sum of up to two 2D Gaussian functions with independent correlation parameters. The fit and the residuals are seen in Fig. 6. Using this method, we observed a change in the correlation parameter of the beam during a single fill in 2018 [2]. The correction values are derived using simulated vdM scans.

\section{Luminous region analysis}

For bunch crossings where primary vertex data is available this method can utilise beamimaging, $v d M$, and a combination of offset and $\mathrm{vdM}$ scans as well. At each beam separation value the density of the primary vertices is fitted with a three-dimensional Gaussian function. The obtained fit parameters, for example, the luminous region centres and widths, and the total rate of 


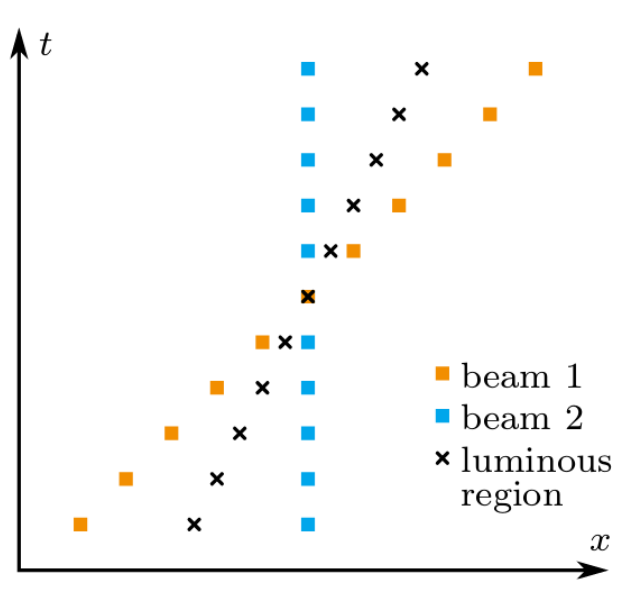

Figure 1: A schematic of one of the four stages of a beam-imaging scan.

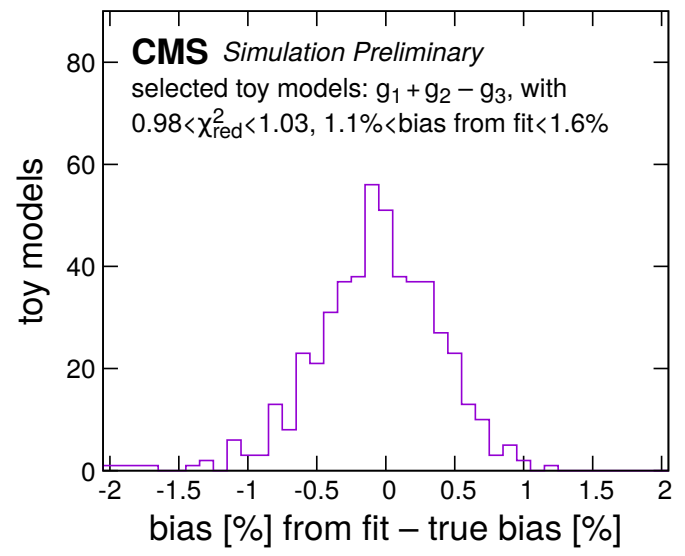

Figure 3: Validation of the beam-imaging analysis using toy models. [6]

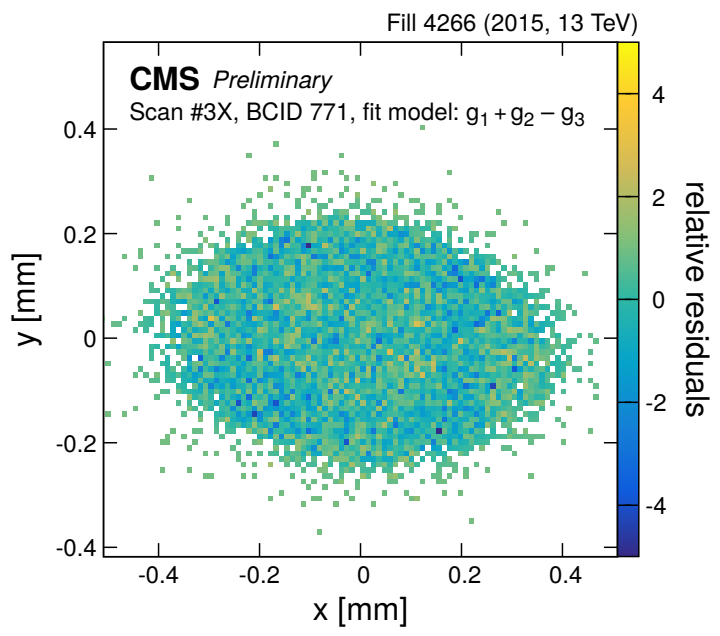

Figure 2: Fit residuals for the best-fit model in 2015 using the beam-imaging analysis. [6]

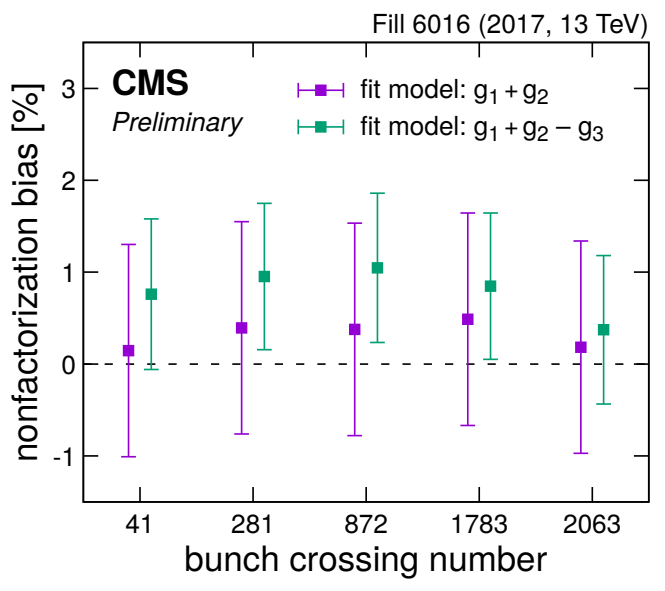

Figure 4: Comparison of vdM bias obtained with different fit models in the beam-imaging analysis in 2017. [6]

one of the luminometers is used to fit the proton density of the bunches. Some of these observables are shown in Figs. 8-11. The density models are built as the sum of 3D Gaussian functions all of which may have a positive or negative weight however the density function is required to be positive at each point in space. The correction values are derived using simulated vdM scans, while the bias and uncertainty of the method is estimated using simulated analyses with randomly generated bunch density functions.

\section{Summary}

In this paper, I briefly introduced the analysis techniques used in CMS to obtain a more precise luminosity measurement and to better understand the systematic uncertainty associated to the 


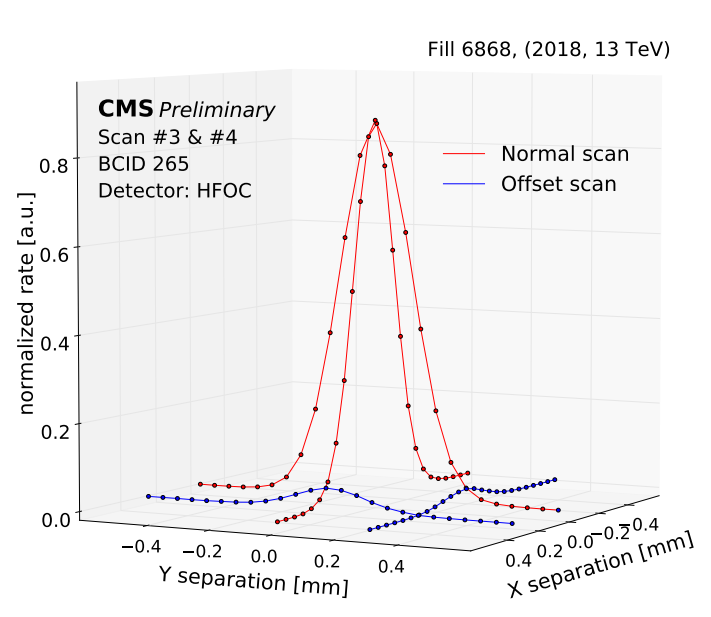

Figure 5: Normalized rates as the function of the beam separations in the $x-y$ plane. The red and the blue points indicate the vdM and the offset data, respectively. [6]
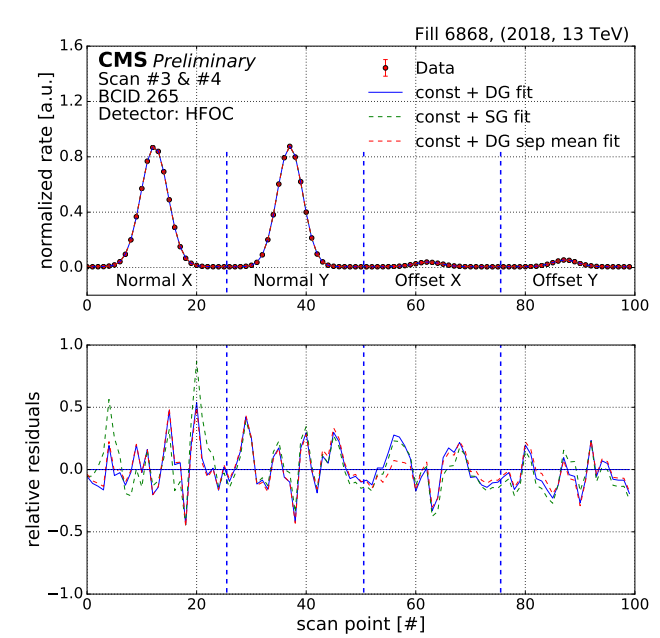

Figure 6: The results of the fit on the vdM and offset data using different models and the residuals. SG and DG stands for 2D single- and doubleGaussian functions, respectively. [6]

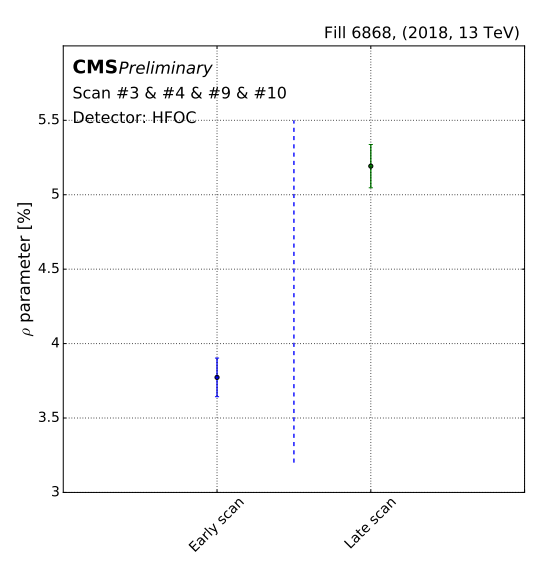

Figure 7: The change of the correlation parameter over the course of a 12 hour scan in 2018 as observed using offset scans. [6]

factorisability of the bunch proton densities in the $x$ and $y$ directions.

\section{References}

[1] CMS Collaboration, The CMS experiment at the CERN LHC, JINST 3 (2008) S08004 [DOI:10.1088/1748-0221/3/08/S08004]

[2] CMS Collaboration, CMS luminosity measurement for the 2018 data-taking period at $\sqrt{s}=13 \mathrm{TeV}$, CMS-PAS-LUM-18-002

[3] P. Major on behalf of the CMS Collaboration, Precision luminosity measurement of proton-proton collisions at the CMS experiment in Run 2, in these proceedings 

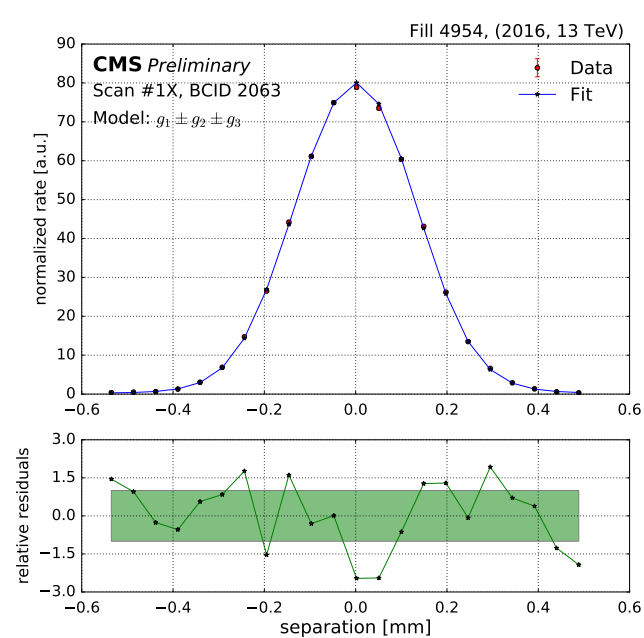

Figure 8: Normalized rates in the $x$ scan. Data in red and fitted model in blue. [6]
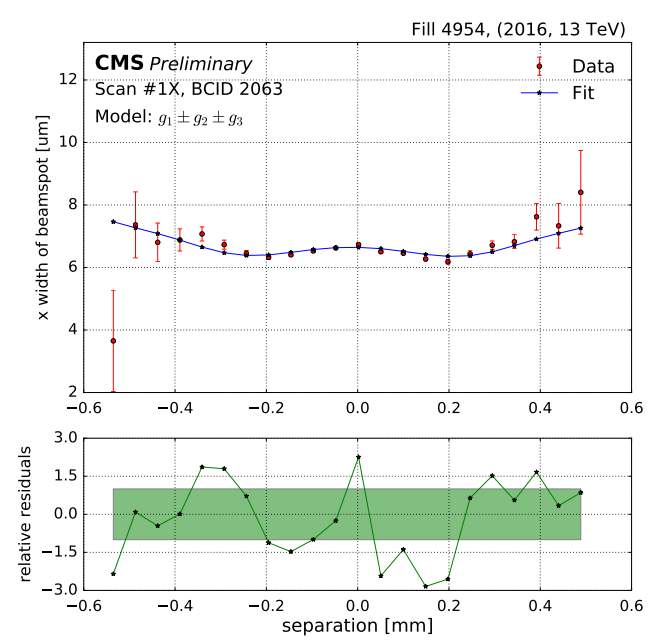

Figure 10: The $x$ width of the luminous region in the $x$ scan. Data in red and fitted model in blue. [6]
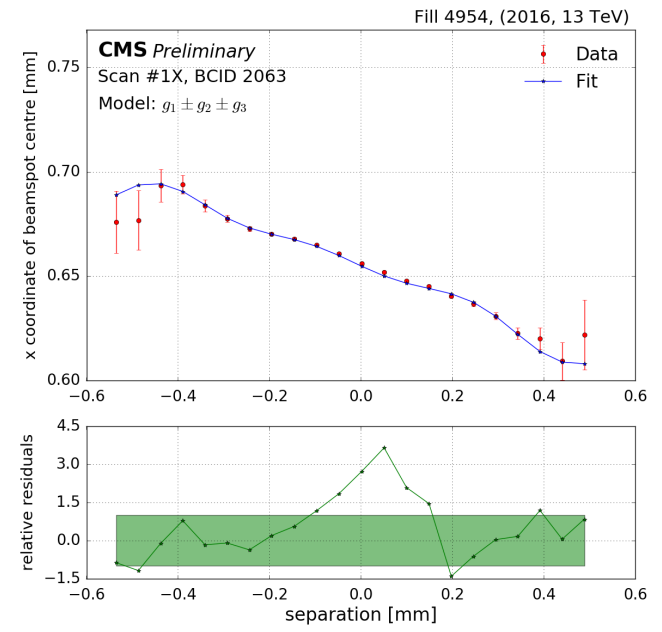

Figure 9: The $x$ coordinate of the luminous region center in the $x$ scan. Data in red and fitted model in blue. [6]
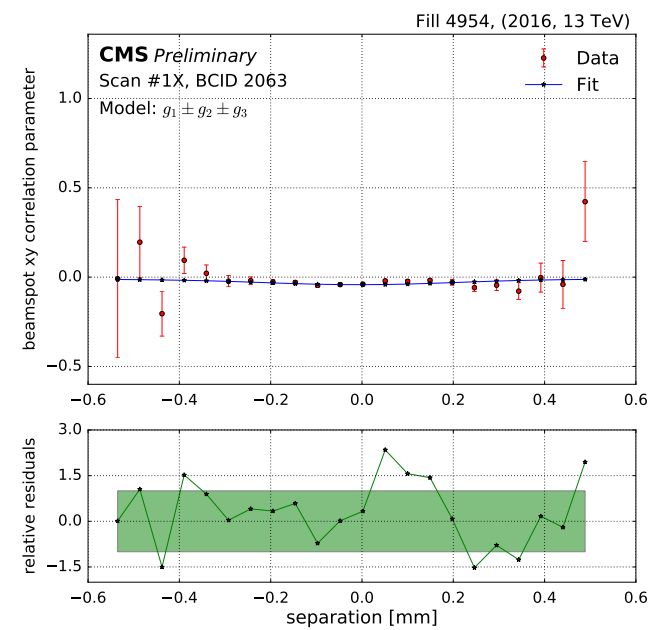

Figure 11: The correlation parameter of the luminous region in the $x$ scan. Data in red and fitted model in blue. [6]

[4] S. van der Meer, Calibration of the effective beam height in the ISR, Technical Report CERN-ISR-PO-68-31, 1968.

[5] M. Klute, C. Medlock, and J. Salfeld-Nebgen, Beam imaging and luminosity calibration, JINST 12 (2017) P03018 [doi:10.1088/1748-0221/12/03/P03018]

[6] CMS Collaboration, Nonfactorisation in Van der Meer scans in Run 2, CMS DP-2019/019 